\title{
Cervicothoracic lipoblastoma: three case reports and review of literature
}

\author{
Alaa Ghallab ${ }^{1 *}$ (D), Mohamed Elmahmoud ${ }^{2}$, Majed Alhadad ${ }^{3}$, Khalil Albatniji', Ameen Alsaggaf ${ }^{1}$, A. Gawad Elhendy ${ }^{2}$, \\ Alam Alshafi ${ }^{3}$ and Abdulwahab S. Aljubab ${ }^{3}$
}

\begin{abstract}
Background: Lipoblastoma is a rare benign mesenchymal tumor of embryonal fat affecting mainly children below 3 years of age. It presents usually as a painless rapidly growing soft lobular mass in the extremities, trunk, and less frequently in the head-neck region. Preoperative imaging is used to assess the extent of disease and aid surgical planning. Complete surgical excision without injury to surrounding vital structures is the treatment of choice.

Case presentation: We report three interesting lipoblastoma cases: mediastinal lipoblastoma with airway compression and ipsilateral diaphragmatic eventration, neck lipoblastoma with intrathoracic extension, and huge thoracic inlet lipoblastoma with compression of common carotid and Lt subclavian arteries, brachial plexus, and ipsilateral diaphragmatic eventration. Complete excision of lipoblastoma mass was done via neck incision in two cases, and the third case required thoracoscopic excision of intrathoracic remnant 6 months later. All three patients had an excellent outcome.

Conclusions: Management of cervicothoracic lipoblastoma is a surgical challenge due to the potential for rapid growth and extension to different fascial planes; however, successful excision can be achieved via a neck approach. Complete surgical excision is essential to prevent local recurrence and improve the outcome.
\end{abstract}

Keywords: Lipoblastoma, Mediastinal, Surgical excision, Prognosis

\section{Background}

Lipoblastoma was first described by Kirshnan et al. in $1926[1,2]$ is a benign tumor of embryonal fat affecting infancy and early childhood with $90 \%$ occurring before 3 years of age and $40 \%$ in the first year of life $[1,3]$. It can arise almost anywhere within the soft tissue. It usually presents with painless swelling in extremity, trunk and less commonly in head and neck [3, 4]. Lipoblastoma symptoms vary depending on the site and size of the tumor [2,3]. Preoperative imaging is not diagnostic but it is used to assess the extent of disease and aid surgical planning [3]. Complete surgical excision is essential for good outcome $[2,5]$.

\footnotetext{
* Correspondence: alaaghallab1969@hotmail.com

'Department of Pediatric Surgery, King Fahd Armed Forces Hospital, PO Box 11259, Jeddah, Kingdom of Saudi Arabia

Full list of author information is available at the end of the article
}

\section{Case report}

\section{Case 1}

A 3-year-old boy presented to our clinic with a history of painless left progressive supraclavicular swelling over 1 year, with no other associated symptoms. On examination, he was comfortable with normal vital signs. The left supraclavicular swelling is oval in shape, $5 \times 4 \mathrm{~cm}$ approximately (Fig. 1a).

Left upper limb examination showed decreased sensation and motor power with weak wrist and finger extension. Additionally, he had weak brachial, radial, and ulnar pulses on the left side. His general blood profile and tumor markers were normal.

Neck and chest $\mathrm{x}$-ray showed tracheal deviation to the right side (Fig. 1b). CT scan showed fat density left supraclavicular mass extending to the upper mediastinum with no calcification or infiltration to surrounding structures. It was encapsulating the left subclavian artery 


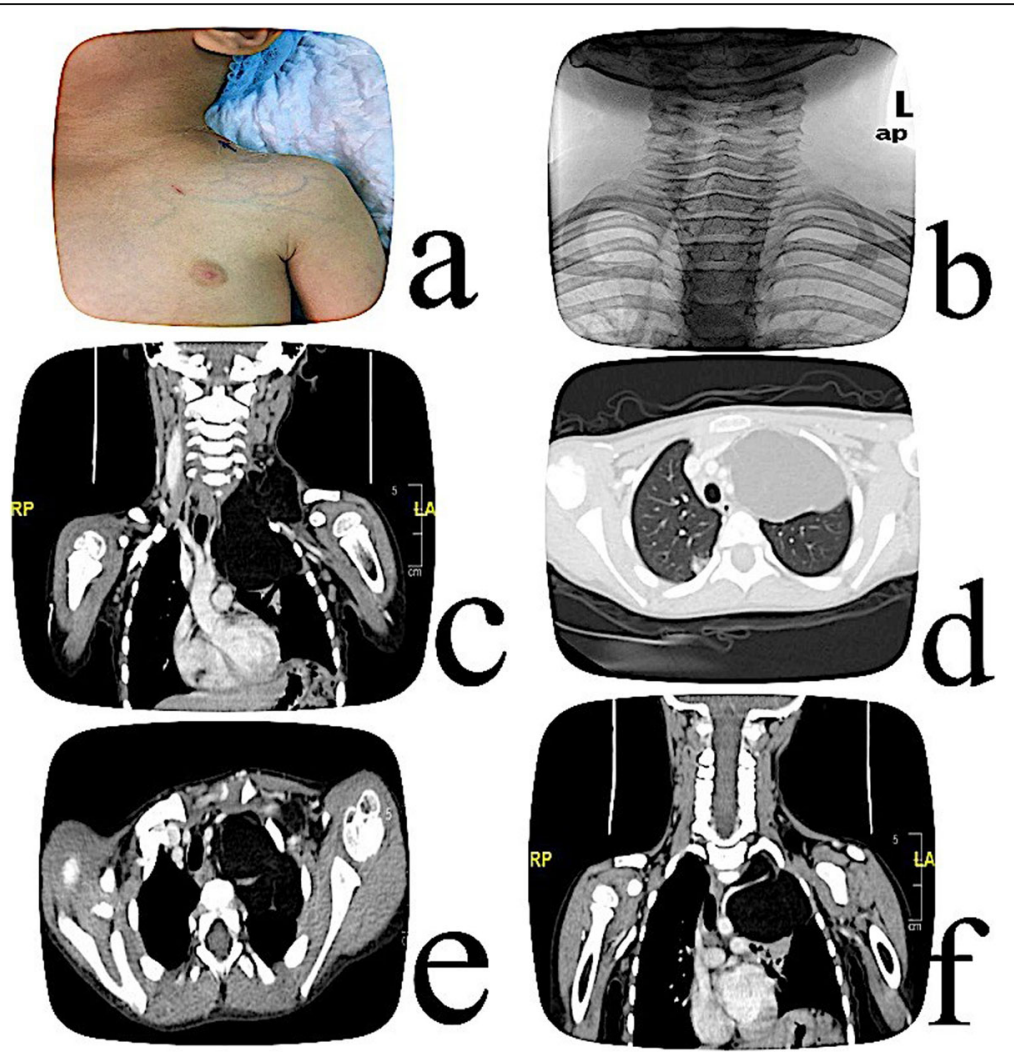

Fig. 1 a Neck mass with dilated superficial veins. $\mathbf{b}$ CXR tracheal deviation to the right side. $\mathbf{c}$ Lt neck mass with intrathoracic extension measuring about $7 \times 8 \mathrm{~cm}$. $\mathbf{d}$ The mass pushed upper mediastinum to the left. e, $\mathbf{f}$ The left subclavian artery was encapsulated by the fatty mass

and compressing the left common carotid artery and left internal jugular vein. The left side of the diaphragm was elevated raising the suspicion of left phrenic nerve compression. It was felt to be a benign fatty tumor (Fig. $1 \mathrm{c}$, d, e, f).

MRI showed a cervicothoracic benign lipomatous mass at the left thoracic inlet as shown in Fig. $2 \mathrm{a}$ and b. The patient underwent surgery via a left supraclavicular transverse incision $1 \mathrm{~cm}$ above the clavicle. The mass stretched the brachial plexus cephalad anteriorly, and the left subclavian vein anteriorly (Fig. $2 \mathrm{c}, \mathrm{d}, \mathrm{e}$ ). Both sharp and blunt dissection were carried out through the incision down to the thoracic inlet where the encapsulated left subclavian artery was freed (Fig. 3). We dissected the intrathoracic portion of the mass via the same incision until it was separated from surrounding structures and then extracted through the wound.

Postoperative course was uneventful, and the patient was discharged home on the second postoperative day with regular physiotherapy. At 6 months follow-up, he had regained full sensory and motor power of the left upper limb. There were no signs of recurrence. Histopathological examination of the left cervical and thoracic lesions confirmed mature lipomatous neoplasm, consistent with lipoblastoma (Fig. 2f).
Follow-up MRI showed a residual mass attached to the inferior border of the left clavicle, measuring $2.3 \times$ $2.3 \times 3.2 \mathrm{~cm}$ compared to $5.1 \times 8.2 \times 7.0 \mathrm{~cm}$ in the preoperative examination. The lesion is seen in the left lung apex causing focal mass effect on the left upper lobe with no sign of invasion (Fig. $3 \mathrm{a}$ and $\mathrm{b}$ ).

The patient was consented for thoracoscopic excision (VATS) of the lipoblastoma remnant, and thoracoscopic excision of the mass was performed by LigaSure. The patient did well and was discharged home on the second postoperative day. Outpatient clinic follow-up showed he was symptom-free, and histopathology confirmed predominantly mature adipose tissue, consistent with lipoma and one benign lymph node. Follow-up chest $\mathrm{x}$ ray undertaken a year later showed slight elevation of the left diaphragm (Fig. 3 a).

\section{Case 2}

A 2-year-old boy was referred to our hospital with painless neck swelling for 7 months increasing in size with no other associated symptoms.

All laboratory investigations were normal, and US showed left supraclavicular mass extending to the upper chest. CT showed left supraclavicular heterogeneous soft 

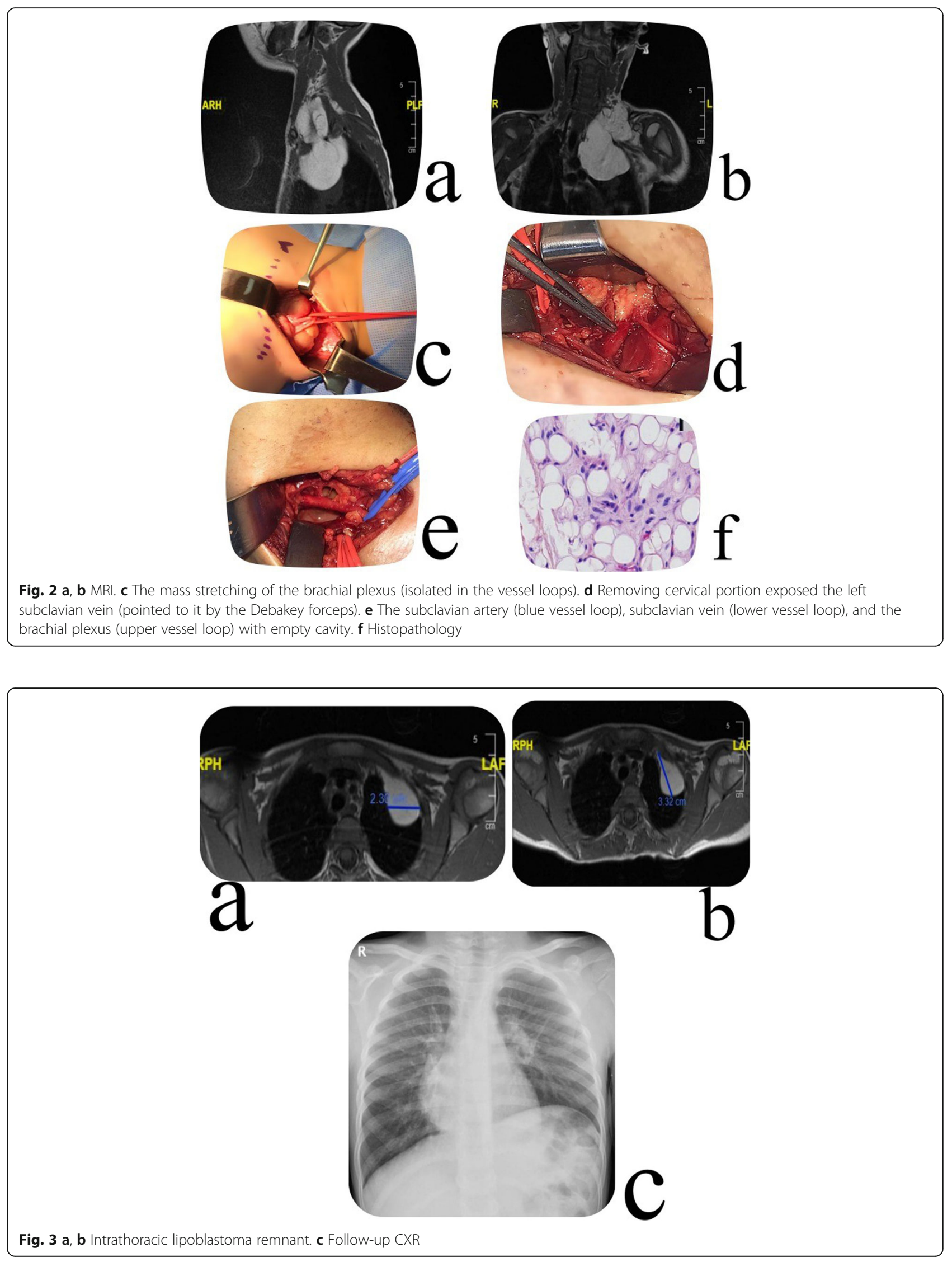
tissue mass $4.8 \times 3.1 \times 3.3 \mathrm{~cm}$, invading between the ribs with extension into the thorax and lung apex (Fig. 4c).

MRI showed a heterogeneous mass in the supraclavicular area extending to the upper chest between the first and second rib (Fig. 4d). True cut biopsy showed fatty adipose tissue tumors of benign nature.

Complete surgical excision was done via transverse supraclavicular incision with removal of thoracic extension by blunt and sharp dissection using bipolar diathermy with preservation of accessory nerve, subclavian vessels, and brachial plexus (Fig. 4a). Histopathology examination confirmed lipoblastoma. Patient had smooth recovery and was discharged home on the second postoperative day. He was doing well at his 6 month follow-up visit.

\section{Case 3}

A 2-year-old boy presented with shortness of breath and cough for 1 month. He had a prior history of intermittent wheeze labeled as asthma and treated with salbutamol nebulization as required. On examination, his vital signs were within normal limits apart from mild tachypnea with reduced air entry at the left lung apex. There was a slight soft bulge at the root of the neck in the extended position. Chest $\mathrm{x}$-ray revealed eventration of $\mathrm{Lt}$ diaphragm reaching up to the 7 th rib, the trachea was deviated to the right (Rt) side, and there was a Lt upper chest opacity.

CT scan showed a $5.1 \times 7.2 \mathrm{~cm}$ fatty mass extending from root of Lt neck to the superior mediastinum (more on the Lt side). There was displacement of the trachea towards the RT side with moderate narrowing of the tracheal lumen, which was most severe at the level of the clavicle where the transverse diameter measured $3 \mathrm{~mm}$. The mass displaced the common carotid artery anteriorly and the Lt subclavian artery posteriorly. It was in direct contact with the posterior part of the aortic arch.

MRI revealed lobular high-intensity tumor equivalent to subcutaneous fat, no solid-enhancing component with complete encasement of the Lt common carotid and subclavian arteries, and it is abutting the posterior aspect of the aortic arch. Percutaneous CT-guided biopsy using $17 / 18$-gauge needle was performed, and pathology report revealed mature fatty tissues.

We decided on a neck exploration via transverse incision at the root of the neck and to proceed for thoracotomy if needed.

Division of Lt sternocleidomastoid muscle exposed the bulging mass which was extending deep to the neck strap muscles and firmly attached to the anterior

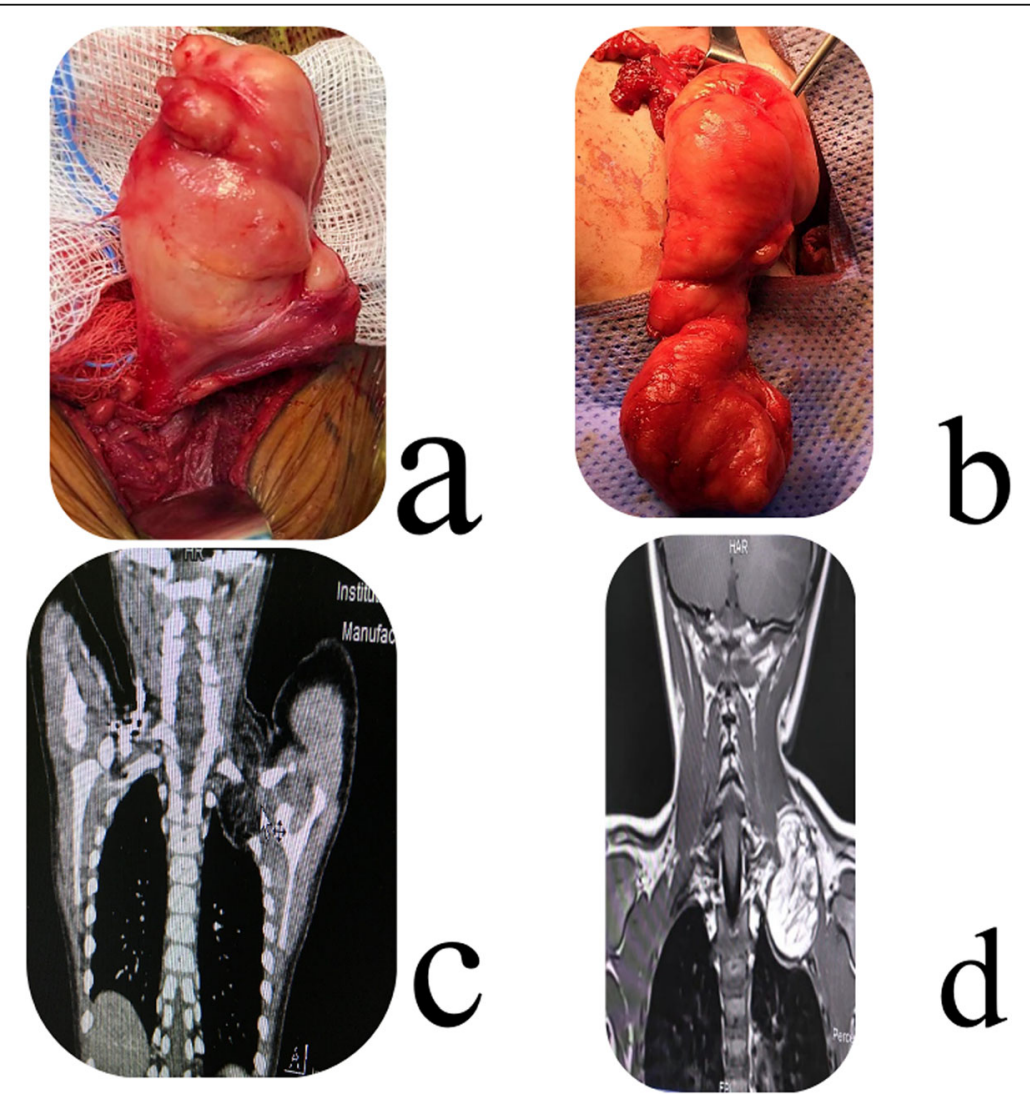

Fig. 4 a Mass delivered to wound. b $11 \times 4 \times 5.5 \mathrm{~cm}$, smooth, lobular, yellow-tan mass, weighed $90 \mathrm{~g}$. c CT neck and chest. d MRI neck and chest 
superior mediastinum. The mass was bluntly retracted into the neck, and a meticulous dissection by LigaSure was carried out until complete removal of the mass (Fig. $4 \mathrm{~b})$. The phrenic nerve was not encountered during surgery.

Microscopic examination revealed that thin pseudocapsule, lobules of lipoblasts, mature adipocytes and myxoid stroma with plexiform vascular pattern, entrapped skeletal muscle, and the lobules were separated by cellular fibrous septa.

Postoperative course was uneventful. He was followed up for 2 years with no signs of recurrence, but Lt diaphragmatic eventration persisted.

\section{Discussion}

Lipoblastoma is a rare benign soft tissue tumor of embryonal fat affecting infancy and early childhood with a male to female ratio of 3:1 [3]. Chung and Enzinger in 1973 categorized lipoblastoma into two types: wellcircumscribed lipoblastoma, a superficial and encapsulated lesion (about $70 \%$ of cases), and lipoblastomatosis, a non-capsulated, diffuse, multicentric, and deeply located with infiltrative growth pattern (about 30\% of cases) $[3,4]$.

It can arise almost anywhere within the soft tissue; the commonest site is extremity, followed by trunk, head, and neck [3, 4]. Other rarer locations include the omentum, scrotum, labia, inguinal, perineum, retroperitoneum, thoracic wall, heart, lungs, and mediastinum $[2,4$, 5]. Mediastinal localization constitutes only 1 to $3 \%$ of all cases, and lipoblastoma is more common (94\%) than lipoblastomatosis in this location [5].

Lipoblastoma symptoms vary depending on the site and size of the tumor, and despite its benign nature, it may become symptomatic by compressing adjacent organs and tissues [2,3]. It presents usually as a painless rapidly growing soft lobular mass in the extremities and trunk [3, 4]. Mediastinal, pleural, pulmonary, and lower neck lipoblastomas can present with dyspnea, wheeze, cough, stridor, and life-threatening asphyxia due to airway compression, vessel compression, superior vena cava syndrome, chest infection, neck swelling, chest wall protrusion, and neurological symptoms resulting from spinal cord compression $[2,5]$. Mesenteric and retroperitoneal lipoblastoma can present with abdominal pain, vomiting, diarrhea, and anorexia [3]. There are few reported cases of huge thoracic inlet lipoblastoma presenting with symptoms predominantly due to mass effect $[3,5]$.

In our mediastinal case, the patient presented with recent onset of cough and tachypnea due to upper airway compression. He had a more chronic history which was labeled as possible asthma and in hindsight was due to mass effect. To our knowledge, phrenic nerve involvement with mediastinal lipoblastoma has been reported in two cases in the literature, and diaphragmatic eventration was present in one of them [6,7].

In our cases, we hypothesize that the long-standing mass compression of LT phrenic nerve resulted in ipsilateral eventration. We were unable to visualize the $\mathrm{Lt}$ phrenic nerve due to the cervical approach and maintained dissection close to the mass.

Both CT and MRI are excellent tools for determining the origin of the tumor, its anatomical extent, composition of the lesion, and the proportion of adipose tissue versus myxoid stroma as well as the presence of internal fibrous tissue, vascular bundles, and soft tissue nodules. Myxoid components are more prominent in infants and young children, whereas fat tissue is predominant in older children $[3,8]$.

The presence of soft tissue components and cystic changes in a predominantly fatty mass should alert the possibility of lipoblastoma [2,3].

Most teratoma and dermoid cyst contain cystic areas and calcification in addition to soft tissue and fatty elements which is not a feature of lipoblastoma [3]. It is imperative to differentiate benign from malignant lipomatous tumors because their prognosis and management are different [2, 3].

Complete surgical resection of lipoblastoma is essential to relieve the patient's symptoms, establish the correct diagnosis, and prevent local recurrence $[2,5,6]$. Clinical outcome depends on completeness of resection as various studies have not reported any recurrences in completely resected tumors with median follow-up period of 40 months [9]. In the literature, there is a very wide range of estimates of the rate of recurrence of resected lipoblastoma's ranging from 0 to $80 \%[4,6,9]$. This is likely to be due to the heterogenous populations with varying types of lipoblastomas in different sites affecting the completeness of the resection and subsequent recurrence rate.

Injury to major structure during surgery should be avoided as it is a benign tumor $[2,4,6]$. Most reported cases of mediastinal lipoblastoma with neck extension were operated via thoracotomy [6,7]. In our cases, we were able to remove the tumor completely through a cervical approach in two patients. We feel consideration should be made to approach these tumors via neck incision. We excised a huge thoracic inlet mass via cervical approach in a 3-year-old boy, and he regained full sensory and motor power of the left upper limb. Postoperative MRI follow-up showed a small infraclavicular remnant which was excised thoracoscopically, and histological examination confirmed lipoma. Follow-up chest x-ray showed marked improvement of ipsilateral diaphragmatic eventration in 
the thoracic inlet case and persistence of it in the mediastinal case.

Lipoblastoma is characterized by the proliferation of mature and immature fat cells with mesenchymal precursors and lipoblasts at varied stages of differentiation with plexiform vascular pattern and abundant myxoid stroma organized in lobules by fibrous septa $[2,3,8]$. Tumor cells display the absence of significant nuclear atypia and pleomorphism with an extremely low mitotic rate $[2,3]$. The diffuse variant shows a less-pronounced lobular pattern $[3,8]$.

Sometimes, the histological diagnosis is difficult because of the close resemblance with myxoid, round cell, and well-differentiated liposarcoma $[1-3,8]$. The helpful clues in differentiation of liposarcoma and lipoblastoma are liposarcoma being rare in children under 10 years, lack of lobulation, variable growth pattern, and increased nuclear atypia $[3,8]$.

In our cases, the patient's age, absence of mitosis, and atypical nuclei, and molecular cytogenetic analysis helped in making the correct diagnosis in the third case.

Histological examination is diagnostic of lipoblastoma; in borderline cases, cytogenetics may be useful to establish a definitive diagnosis $[2,3,8]$.

Most of lipoblastoma tumors grow slowly but steadily with a potential for local invasion [4, 5]; however, the prognosis is good with no reported metastasis nor malignant transformation $[1,2]$.

The long-term outcome of the thoracic inlet lipoblastoma in children is not as well-defined as in adults with only a few pediatric case reports [2]. Therefore, long-term follow-up with regular imaging is essential in children with multiple recurrences or incomplete tumor resection, and re-excision is the treatment of choice [2-4].

\section{Conclusions}

Lipoblastoma is a rare benign tumor of embryonal fat that occurs more commonly in male infants and children less than 3 years of age. We present rare cases of cervicothoracic lipoblastomas with ipsilateral diaphragmatic eventration and upper airway compression. Preoperative imaging is not diagnostic but is beneficial to assess the extent of disease and aid surgical planning. Mediastinal lipoblastoma is a surgical challenge due to the potential for rapid growth and extension to different fascial planes; however, successful excision can be achieved via a neck approach. Complete surgical excision without injury to surrounding vital structures is the treatment of choice as it yields an excellent prognosis. There is a tendency for these lesions to recur despite presumed complete excision. Therefore, follow-up for a minimum of 5 years is recommended.

\section{Abbreviations}

CT: Computed tomography; MRI: Magnetic resonance imaging; Rt: Right; Lt: Left

\section{Acknowledgements}

Dr MM Shalaby for editing the figures

\section{Authors' contributions}

All authors contributed equally in the writing of this manuscript. MA, AE, and KA collected the data and managed the first case. AG and AA collected the data and managed the second case. AAG, AA, and MA collected the data and managed the third case. The authors read and approved the manuscript and ensure that this is the case.

\section{Funding}

None

Availability of data and materials

The datasets used during the current study are available from the corresponding author on reasonable request.

\section{Declarations}

\section{Ethics approval and consent to participate}

Approval was obtained from the Research Ethics committee at our three centers. Parents of each patient were informed about all steps in the procedure.

Approval code: REC 437

\section{Consent for publication}

A written consent for publication was obtained from parents.

Competing interests

The authors declare that they have no competing interest.

\section{Author details}

${ }^{1}$ Department of Pediatric Surgery, King Fahd Armed Forces Hospital, PO Box 11259, Jeddah, Kingdom of Saudi Arabia. ${ }^{2}$ Department of Pediatric Surgery, Prince Sultan Military Medical City, Riyadh, Kingdom of Saudi Arabia.

${ }^{3}$ Department of Pediatric Surgery, King Fahd Medical City, Riyadh, Kingdom of Saudi Arabia.

Received: 15 May 2021 Accepted: 9 July 2021

Published online: 01 November 2021

References

1. Kirshnan J, Hathiramani V, Hastak M, Redkar RG. Myxoid lipoblastoma. Indian Pediatr. 2013;50:603-5.

2. Spinelli C, Costanzo S, Severi E, Giannotti G, Massart FA. Thoracic wall lipoblastoma in a 3 month old infant: a case report and review of the literature. J Pediatr Hematol Oncol. 2006;28(9):594-600. https://doi.org/10.1 097/01.mph.0000212977.13749.c3.

3. Bruyeer E, Lemmerling M, Poorten W, Sciot R, Hermans R. Paediatric lipoblastoma in the head and neck: three cases and review of literature. Cancer Imaging. 2012;12(3):484-7.

4. Chung EB, Enzinger FM. Benign lipoblastomatosis. An analysis of 35 cases. Cancer. 1973;32(2):482-92. https://doi.org/10.1002/1097-0142(197308)32:2< 482::AID-CNCR2820320229>3.0.CO;2-E.

5. Hudsona SA, Lacsona GA, Dicken JB. Benign giant mediastinal lipoblastoma. J Pediatr Surg Case Rep. 2019;40:38-42. https://doi.org/1 0.1016/j.epsc.2018.10.013.

6. Mutlu M, Yaris N, Imamoglu M, Kosucu P, Turgutalp H. Mediastinal lipoblastoma causing diaphragmatic eventration: a case report and review of the literature. J Pediatr Hematol Oncol. 2009;31:346-8 https://doi.org/10.1 097/MPH.0b013e31818e5354.

7. Irgau I, McNicholas WK. Mediastinal lipoblastoma involving the left innominate vein and the left phrenic nerve. J Pediatr Surg. 1998;33(10): 1540-2. https://doi.org/10.1016/S0022-3468(98)90493-6.

8. Mentzel T. Lipoblastoma and lipoblastomatosis: a clinic-pathological study of 14 cases. Histopathology. 1993;23(6):527-33. https://doi.org/10.1111/j.13 65-2559.1993.tb01238.x. 
9. Abdulghafar J, Ahmed Z, Tariq MU, Kayani N, , Uddin N. Lipoblastoma : a clinicopathologic review of 23 cases from a major tertiary care center plus detailed review of literature, BMC research notes. 2018;11(1):1-6.

\section{Publisher's Note}

Springer Nature remains neutral with regard to jurisdictional claims in published maps and institutional affiliations.

Submit your manuscript to a SpringerOpen ${ }^{\mathcal{O}}$ journal and benefit from:

- Convenient online submission

- Rigorous peer review

- Open access: articles freely available online

- High visibility within the field

- Retaining the copyright to your article

Submit your next manuscript at $\boldsymbol{\wedge}$ springeropen.com 